This item was submitted to Loughborough's Research Repository by the author.

Items in Figshare are protected by copyright, with all rights reserved, unless otherwise indicated.

\title{
EBG enhanced broadband dual antenna configuration for passive self- interference suppression in full-duplex communications
}

PLEASE CITE THE PUBLISHED VERSION

https://doi.org/10.23919/EuRAD.2018.8546551

PUBLISHER

IEEE @ EUMA

VERSION

AM (Accepted Manuscript)

LICENCE

CC BY-NC-ND 4.0

\section{REPOSITORY RECORD}

Deo, Prafulla, Dariush Mirshekar-Syahkal, and Gan Zheng. 2019. "EBG Enhanced Broadband Dual Antenna Configuration for Passive Self-interference Suppression in Full-duplex Communications”. figshare. https://hdl.handle.net/2134/36746. 


\title{
EBG Enhanced Broadband Dual Antenna Configuration for Passive Self-Interference Suppression in Full-Duplex Communications
}

\author{
Prafulla Deo $^{\# 1}$, Dariush Mirshekar-Syahkal ${ }^{\# 2}$, and Gan Zheng*3 \\ \#School of Computer Science and Electronic Eng., University of Essex, Colchester CO4 3SQ, UK \\ "School of Mech., Elect. and Manu. Eng., Loughborough University, Loughborough LE11 3TU, UK \\ ${ }^{1}$ pdeo@essex.ac.uk, ${ }^{2}$ dariush@essex.ac.uk
}

\begin{abstract}
A full-duplex system is realised using dual EBG isolated rectangular spiral antennas and its performance is compared with the same full-duplex system using a circulator and a single spiral antenna element. The new antenna system consists of two antennas one with RHCP and the another one with LHCP implemented on a single substrate. Two columns of EBG is placed between the two antennas to improve the isolation. At the operating frequency of $3.2 \mathrm{GHz}$, the antenna configuration has nearly $31 \mathrm{~dB}$ isolation. For the identical baseband input power, the full-duplex system utilising dual spiral antenna configuration exhibits $9 \mathrm{~dB}$ higher isolation than the circulator based full-duplex system.
\end{abstract}

Keywords - spiral antenna, full-duplex system, antenna isolation, self-interference cancellation.

\section{INTRODUCTION}

With more and more data-intensive services being introduced in the wireless domain, the amount of data traffic over the wireless networks is increasing exponentially whereas the availability of wireless spectrum remains limited. To meet the demands for high data rate communications, new technologies such as Long Term Evolution (LTE) and LTEAdvanced have been introduced. Despite the fact that these technologies are capable of providing high-speed, highcapacity wireless services, it relies on the systems deploying half-duplex radios. Half-duplex radios, transmit and receive signals in two separate or orthogonal channels. Recently, progress has been made in in-band full-duplex (FD) wireless systems in which both the uplink and downlink transmission is carried out over the same frequency channel. This ability to both transmit and receive the signals at same frequency channel offers the potential to double the spectral efficiency of the wireless network.

The prime challenge in utilizing FD is to isolate and suppress the strong in-band leakage from the transmitter to the receiver also known as self-interference (SI). The magnitude of self-interference can be in the range of 50-110 dB higher than the received signal, depending on the separation between the transmitter and receiver. The desired received signal is therefore dominated by the SI necessitating SI cancellation between the transmitter and receiver. In recent literature, several self-interference cancellation techniques have been

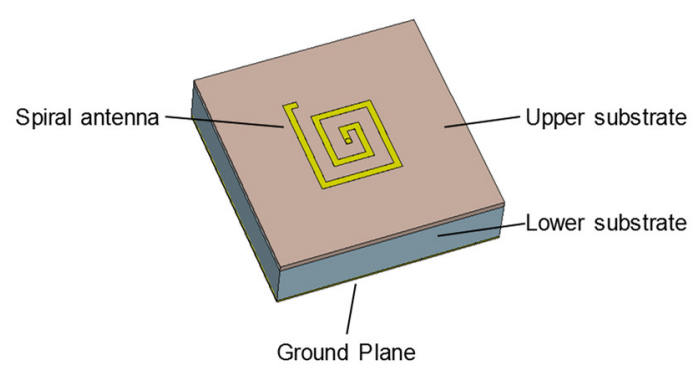

(a)

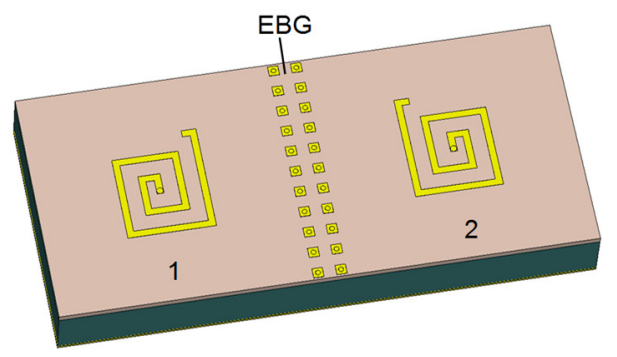

(b)

Fig. 1. (a) Single-arm rectangular spiral antenna and (b) EBG enhanced two rectangular spiral antennas configuration.

studied [1]. It has been shown that using antenna placements, active analog cancellations or by using active digital cancellation, it is possible to reduce the effects of SI [2]. However, to achieve the highest possible SI cancellation, usually, a combination of antenna placements, active analog cancellation, and active digital cancellation is utilised [3].

Isolation between the transmit and receive antennas in an FD radio system is quite important and it is advisable to achieve high isolation over the desired frequency band to reduce the dependability on analog and digital cancellation stages. FD systems without using circulator has been an important topic of research as the usually large port-to-port coupling between two in-band antennas reduces the efficiency 
and increases their correlation due to an undesirable signal path between the antennas [4].

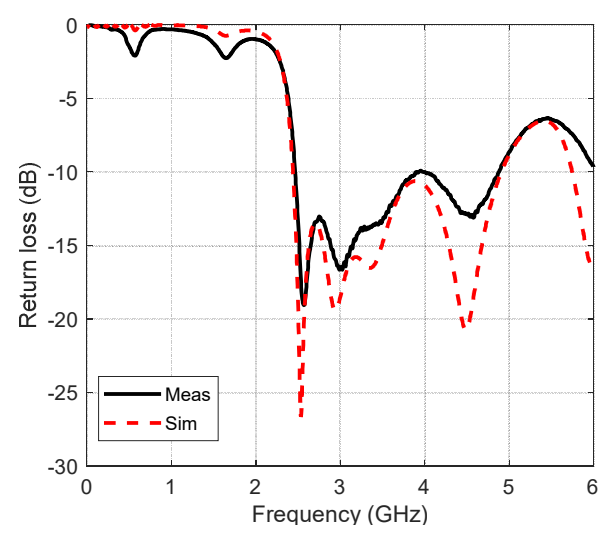

Fig. 2. Reflection coefficient of single rectangular spiral antenna configuration.

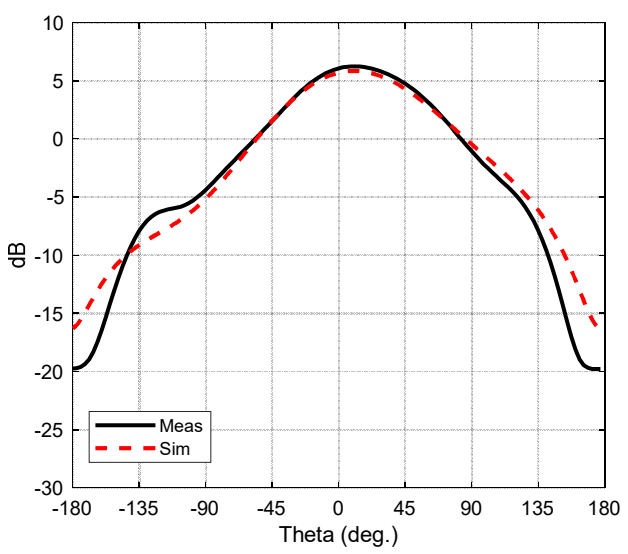

Fig. 3. Rectangular spiral antenna radiation pattern at $3.2 \mathrm{GHz}$.

In this paper, a compact wideband electromagneticbandgap (EBG) enhanced dual antenna configuration is proposed. This dual structure has two spiral antennas, which are orthogonally polarized (LHCP and RHCP) to achieve an effective isolation between the two antenna ports. To improve the isolation further, two columns of EBG is placed between the two antennas. Real-time testing of the FD system utilising the proposed antenna system is performed and the results are compared with circulator based single antenna FD system.

\section{ANTENNA CONFIGURATION}

Fig. 1 shows a single-arm rectangular spiral antenna, similar to the one in [5]. This antenna serves as the radiating element in the proposed dual antenna configuration. The total arm length of the antenna is optimised to generate circularly polarized axial beam pattern at $3.2 \mathrm{GHz}$. The substrate for the antenna is composed of two dielectric layers, stacked together. For the lower substrate, the thickness is $10.55 \mathrm{~mm}$ and dielectric constant is 3.4. For the upper substrate, the thickness is $1.45 \mathrm{~mm}$ and dielectric constant is 3.48 . Thus, the total substrate thickness is $12 \mathrm{~mm}$. The side length of the substrate is $50 \mathrm{~mm} \times 50 \mathrm{~mm}$. The antenna has a ground plane on one

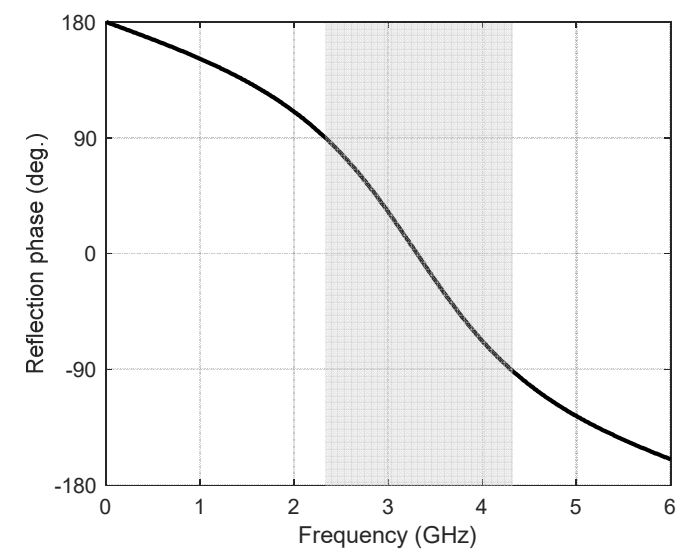

Fig. 4.

Computed reflection phase of an EBG structure. Grey area represents the bandgap region.

side and spiral pattern on the other side. The total length of the spiral arm is $115.5 \mathrm{~mm}$ and its width is $1.4 \mathrm{~mm}$. The antenna is fed at the center from the ground plane side through a $50 \Omega$ coaxial cable.

Two such antennas on the same substrate with opposite sense of windings laid closely, Fig. 1(b), were fabricated. One antenna is for transmission and the other one for the reception.

In Fig. 1(b), Antenna 1 generates the RHCP beams whereas Antenna 2 generates LHCP beams and thus provides some isolation between the two antennas. Both antennas are identical apart from their polarization sense and hence either can be used interchangeably as a transmitting or receiving antenna. With orthogonally polarized antennas, a leakage from the transmitter to the receiver can be reduced despite the short physical distance of the antennas. The dual antenna has overall dimensions of $110 \mathrm{~mm} \times 50 \mathrm{~mm}$. The center to center spacing between the two antennas is $60 \mathrm{~mm}$ and this is chosen to obtain optimized return loss, isolation and radiation patterns. To isolate the SI even further, two columns of mushroom-type EBG having unit cells similar to [6] is inserted. Dimensions of the unit cell are optimised to operate at $3.2 \mathrm{GHz}$ for the same dual layer substrate as of spiral antenna. Each square patch has a side length of $2.1 \mathrm{~mm}$ with a gap of $2.6 \mathrm{~mm}$ between the two consecutive patches. The diameter of the metal via connecting the square patch and ground plane is $1 \mathrm{~mm}$. The EBG structure consisting of $11 \times 2$ array of square metal patches is used in the dual antenna configuration.

The antennas and EBG are designed and analysed using CST Studio Suite software and the antenna measurements are performed using Satimo StarLab Anechoic chamber facility.

\section{RESULTS}

\section{A. Single Spiral Antenna}

The simulated and measured return losses of the proposed spiral antenna is shown in Fig. 2. The $-10 \mathrm{~dB}$ impedance bandwidth covers a broad frequency range of $2.46 \mathrm{GHz}$ to 
4.85 GHz and hence, the antenna offers a large bandwidth of $2.39 \mathrm{GHz}$, Fig. 2. At the operating frequency of $3.2 \mathrm{GHz}$, the

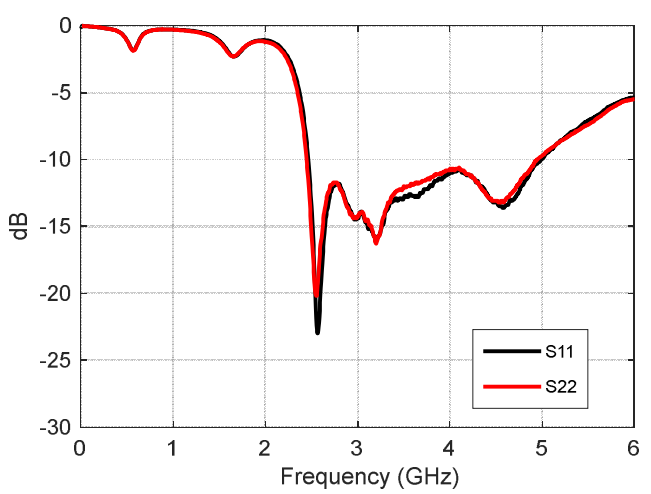

(a)

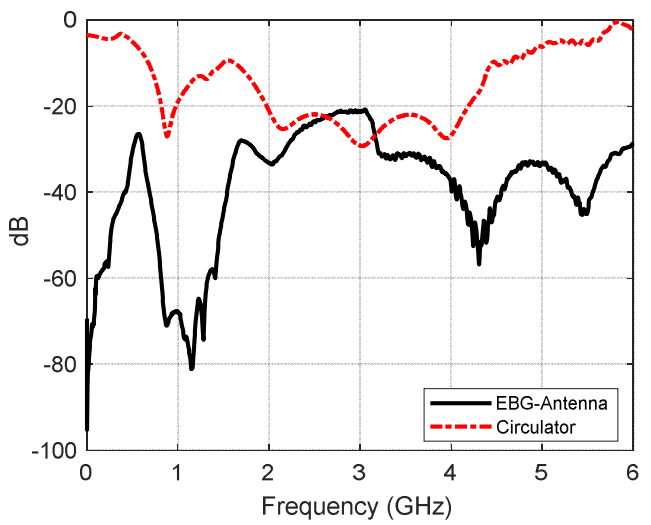

(b)

Fig. 5. Measured (a) reflection coefficient and (b) ports isolation of the dual spiral antenna system using EBG and a commercial circulator.

antenna radiation patterns have a tilt of $\theta_{\max }=11^{\circ}$, Fig. 3. This $11^{\circ}$ tilt is due to the spiral arm asymmetry with respect to the $\mathrm{x} / \mathrm{y}$-axes. The value of the $3-\mathrm{dB}$ beamwidth, Fig. 3 , is approximately $95^{\circ}$. At the operating frequency, the radiation pattern is circularly polarized with an axial ratio of $2.9 \mathrm{~dB}$, and the antenna gain is approximately $6.26 \mathrm{~dB}$.

\section{B. Electromagnetic bandgap structure}

The $11 \times 2$ EBG configuration is shown in Fig. 1(b). The parameters of the periodic EBG were selected and fine-tuned so as to keep the required frequency band in excess of $1.9 \mathrm{GHz}$ (Fig. 4) within the $\pm 90^{\circ}$ reflection phase of the EBG. It has been also shown in previous experiments that using metal vias connecting EBG square patch and ground plane creates a surface wave bandgap which would overlap the frequency band of the EBG reflection phase [6].

\section{Two (dual) rectangular spiral configuration}

Fig. 5(a) shows, the measured $-10 \mathrm{~dB}$ bandwidth of the antenna system is $2.5 \mathrm{GHz}(2.45-4.95 \mathrm{GHz})$ over which the ports isolation varies from $-56.8 \mathrm{~dB}$ to $-21 \mathrm{~dB}$, Fig. 5(b). The isolation achieved with the proposed dual antenna setup is generally better than that offered by the commercially available circulator and one antenna within the common operating bandwidth of $2.5-4 \mathrm{GHz}$. This is shown in Fig. $5(\mathrm{~b})$.

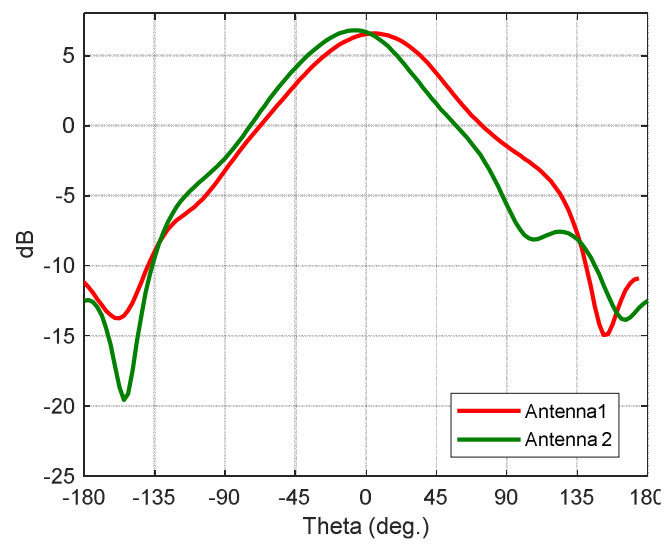

Fig. 6. Measured radiation pattern of Antenna $1 \& 2$ shown in Fig. 1(b) for the elevation cut at $3.2 \mathrm{GHz}$.

At the operating frequency $3.2 \mathrm{GHz}$, isolation between the two antenna ports is $-30.8 \mathrm{~dB}$, whereas for circulator it is $-26 \mathrm{~dB}$. Fig. 6 shows the measured radiation patterns of Antenna 1 and Antenna 2 at $3.2 \mathrm{GHz}$. For Antenna 1, the maximum gain is $6.6 \mathrm{~dB}$ at $\theta_{\max }=5.6^{\circ}$ and for Antenna 2 it is $6.8 \mathrm{~dB}$ at $\theta_{\max }=$ $5.65^{\circ}$. The $3-\mathrm{dB}$ beamwidth and axial ratio for Antenna 1 and Antenna 2 are $85.4^{\circ}$ and $2.9 \mathrm{~dB}, 76^{\circ}$ and $2.8 \mathrm{~dB}$, respectively.

D. Comparison between proposed EBG enhanced dual rectangular spiral antenna configuration and circulator based FD system with one antenna

To investigate the effects of improved isolation in the proposed dual antenna setup, a direct conversion full-duplex system is implemented, Fig. 7, using both the circulator and single spiral antenna, Fig. 1(a), and dual antenna configuration, Fig. 1(b).

For both FD systems, a rectangular pulse train of $10 \mu \mathrm{s}$ (100 KHz) and $95 \mathrm{mV}$ amplitude was used as a baseband signal. The frequency of the local oscillator (LO) is kept at $3.2 \mathrm{GHz}$. The output at the receiver is measured and shown in Fig. 8. It is clear from Fig. 8 that the proposed dual antenna system $(6 \mathrm{mV})$ suppresses the SI better than the circulator based system $(17.5 \mathrm{mV})$. The received power for dual spiral antenna FD system, Fig. 7(b), was around $-54 \mathrm{dBm}$ whereas for the circulator based system Fig. 7(a) it was nearly $-45 \mathrm{dBm}$. Thus the proposed dual spiral antenna configuration provides $9 \mathrm{~dB}$ higher isolation than the circulator based system.

\section{CONCLUSION}

The design and realization of EBG enhanced dual orthogonally polarized broadband rectangular spiral antenna were presented and discussed. The dual antenna structure has two broadband spiral antennas, one with RHCP and another one with LHCP. Two rows of EBG were placed between the two antennas to improve the isolation. At the operating frequency of $3.2 \mathrm{GHz}$, the antenna configuration provides nearly $31 \mathrm{~dB}$ isolation. When used with direct conversion fullduplex system, the proposed antenna configuration provides 
[4] E. Ahmed et al., "Full-Duplex Systems Using Multireconfigurable

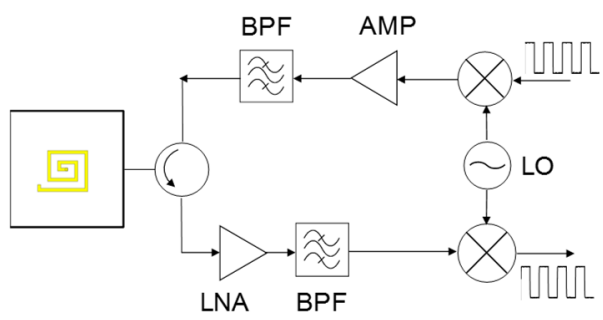

(a)

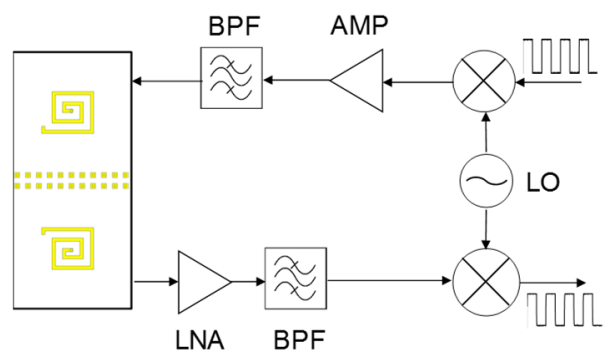

(b)

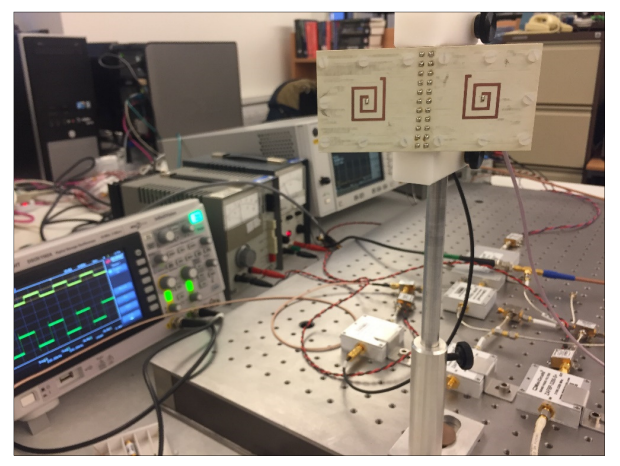

(c)

Fig. 7. Direct conversion full-duplex system using (a) circulator and single spiral antenna (b) EBG enhanced dual spiral antenna and (c) FD system setup for proposed dual antenna configuration.

$9 \mathrm{~dB}$ more isolation when compared with circulator based FD system using a single rectangular spiral antenna.

\section{ACKNOWLEDGMENT}

This work was supported by EPSRC (UK) under grants EP/N007840/1 and EP/N008219/1.

\section{REFERENCES}

[1] A. Sabharwal et al., "In-Band Full-Duplex Wireless: Challenges and Opportunities," IEEE JSAC, vol. 32, no. 9, Oct. 2014, pp. 1637-52.

[2] M. Duarte and A. Sabharwal, "Full-duplex wireless communications using off-the-shelf radios: Feasibility and first results," in Conf. Rec. 44th ASILOMAR Signals, Syst. Comput., Nov. 2010, pp. 1558-1562.

[3] D. Korpi et al., "Advanced self-interference cancellation and multiantenna techniques for full-duplex radios," in Asilomar Conference on Signals, Systems and Computers, Nov. 2013, pp. 3-8.

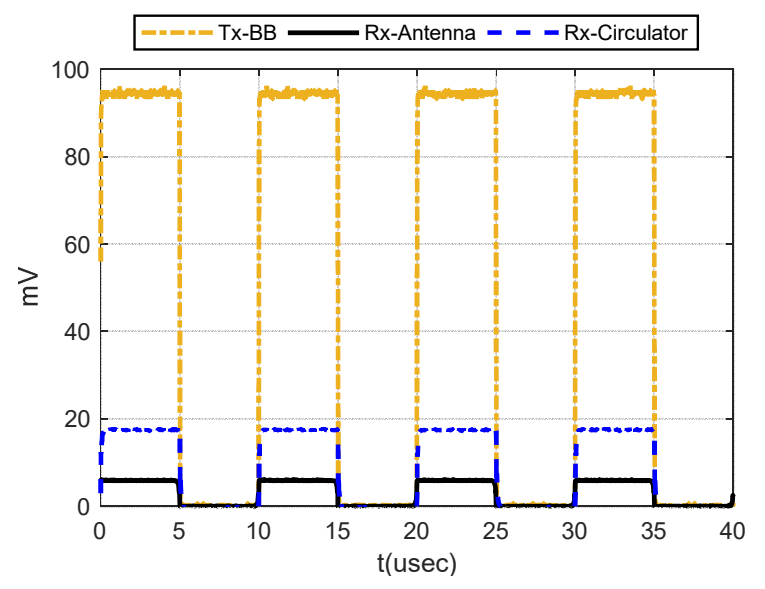

Antennas", IEEE Trans., Wireless Comms. Vol. 14, No. 11, Nov. 2015, pp. 5971-5983.

Fig. 8. Recovered baseband from direct conversion full-duplex system using dual antenna configuration and circulator based system.

[5] H. Nakano et al., "Tilted- and axial beam formation by a single-arm rectangular spiral antenna with compact dielectric substrate and conducting plane", IEEE Trans., Antennas Propag. Vol. 50, No. 1, Jan. 2002, pp. 17-23.

[6] D. Sievenpiper, L. Zhang, R. F. J. Broas, N. G. Alexopolous, and E. Yablonovitch, "High-impedance electromagnetic surfaces with a forbidden frequency band," IEEE Trans. MTT, vol. 41, pp. 2059-2074, Nov. 1999. 